\title{
Aluminatos de cálcio e seu potencial para aplicação em endodontia e ortopedia
}

\section{(Calcium aluminates potential for endodontics and orthopedic applications)}

\author{
L. L. M. F. Pompeu ${ }^{1}$, G. L. Santos ${ }^{1}$, V. C. Pandolfelli ${ }^{2}$ I. R. Oliveira ${ }^{1^{*}}$ \\ ${ }^{1}$ Universidade do Vale do Paraíba - Univap, Av. Shishima Hifumi 2911, S. José dos Campos, SP 12244-000 \\ ${ }^{2}$ Grupo de Engenharia de Microestrutura de Materiais - GEMM, Departamento de Engenharia de Materiais, \\ Universidade Federal de S. Carlos, Rod. Washington Luiz, km 235, C.P. 676, S. Carlos, SP 13565-905 \\ *ivonero@univap.br
}

\begin{abstract}
Resumo
Os materiais de maior uso em Endodontia como retro-obturador ou selador de defeitos na comunicação da raiz com o periodonto lateral (MTA, mineral trioxide aggregate) e em Ortopedia, para fixar implantes e na remodelação óssea perdida (PMMA, polimetilmetacrilato), apresentam respectivamente, algumas propriedades limitantes como: longo tempo de presa e alta temperatura durante a polimerização. Tais limitações indicam a necessidade de alterações em suas composições bem como o desenvolvimento de materiais alternativos visando expandir suas aplicações. Neste contexto, um novo biomaterial a base de cimento de aluminato de cálcio (CAC) tem sido estudado visando preservar as propriedades positivas e aplicações clínicas do MTA e PMMA superando algumas de suas desvantagens. Estudos recentes envolvendo o uso de CAC têm sido baseados em produtos comerciais compostos por uma mistura de fases. Melhorias neste sentido podem ser alcançadas pesquisando as rotas de síntese do CAC visando o adequado balanço entre as fases e o controle das impurezas que podem prejudicar a sua atuação em aplicações em áreas da saúde. A produção de fases de CAC isoladamente foi estudada anteriormente e neste trabalho é apresentada a sua caracterização quanto à temperatura de reação de hidratação; trabalhabilidade/tempo de presa e quanto a influência sobre pH, condutividade iônica e concentração de sólidos da água e diferentes soluções simuladoras de fluido corporal quando em contato com as fases. Os resultados apresentados indicaram as fases $\mathrm{CaO} \cdot \mathrm{Al}_{2} \mathrm{O}_{3}(\mathrm{CA})$ e $\mathrm{CaO} \cdot 2 \mathrm{Al}_{2} \mathrm{O}_{3}\left(\mathrm{CA}_{2}\right)$ como as mais promissoras para as aplicações pretendidas.
\end{abstract}

Palavras-chave: cimento de aluminato de cálcio, temperatura, tempo de pega, $\mathrm{pH}$, condutividade iônica, fluido corporal simulado.

\section{Abstract}

The mostly used material in Endodontics as root-end filling or perforation as well as root canal sealers (MTA, mineral trioxide aggregate) and in orthopedic surgery to affix implants and to remodel bone loss (PMMA, polymethyl methacrylate), present respectively, some limiting properties such as: long setting time and high polymerization temperature. These drawbacks highlight the need of improvements in their compositions, as well as the development of alternative materials aiming to expand their applications. In this context, a novel biomaterial-based calcium aluminate cement (CAC) has been studied in order to keep the positive properties and clinical applications of MTA and PMMA, overcoming some their disadvantages. Recent studies involving the use of CAC are based on commercial products consisting of a mixture of phases. Improvements can be attained by searching the synthesis routes of $C A C$ aiming the proper balance between the phases and the control of impurities that may impair its performance in applications in the areas of health. The production of CAC phases has been previously studied and this article present their characterization based on hydration temperature; working time/setting time and their influence on $\mathrm{pH}$, ionic conductivity and solids concentration of water and different solutions of simulated body fluid in contact with these phases. The results indicated the $\mathrm{CaO} . \mathrm{Al} \mathrm{O}_{3}(\mathrm{CA})$ and $\mathrm{CaO} .2 \mathrm{Al}_{2} \mathrm{O}_{3}\left(\mathrm{CA}_{2}\right)$ phases as the most suitable for the target applications.

Keywords: calcium aluminate cement, temperature, setting time, pH, ionic conductivity, MTA, simulated body fluid.

\section{INTRODUÇÃO}

Os conceitos atuais que norteiam o tratamento endodôntico enfatizam a limpeza e a modelagem do sistema de canais radiculares, bem como a utilização de materiais biocompatíveis e técnicas que propiciam uma obturação tridimensional do mesmo, o que possibilita elevados índices de sucesso na terapia endodôntica. Apesar disto, em determinados casos, em função de processos patológicos, alterações degenerativas, dentes anatomicamente desfavoráveis, impossibilidade de acesso endodôntico e iatrogenias, somente com o auxílio da cirurgia perirradicular o órgão dental pode ser preservado [1]. Nesta cirurgia é realizada a apicectomia, representada pela associação da curetagem com a amputação do ápice radicular, complementada pela obturação retrógrada da raiz. O material de maior destaque como obturador retrógrado é o MTA (Agregado de Trióxido Mineral). Seu uso não se restringe ao processo de retrobturação, também é usado em capeamentos pulpares diretos, reparações 
de perfurações radiculares ou de furcas e defeitos de reabsorção perfurantes $[2,3]$. O MTA se apresenta como um pó de finas partículas hidrofílicas que endurecem após hidratação. $\mathrm{O}$ pó é composto principalmente por silicato tricálcico, aluminato tricálcico, óxido tricálcico e óxido de silicato, além de pequenas quantidades de outros óxidos minerais e da adição de óxido de bismuto, responsável pela radiopacidade do material $[2,3]$. O mecanismo de ação do MTA é descrito pela conversão do óxido de cálcio presente no pó em hidróxido de cálcio ao realizar-se a preparação da pasta com a água. O hidróxido de cálcio, por sua vez, em contato com os fluidos tissulares se dissocia em íons cálcio e hidroxila. Os íons cálcio reagem com o gás carbônico dos tecidos dando origem a granulações de calcita. A própria alcalinidade do meio estimula células do tecido conjuntivo a secretar uma glicoproteína, a "fibronectina", a qual em contato com os cristais de calcita favorece o depósito de colágeno tipo I. A continuidade desse processo, agora com íons $\mathrm{Ca}^{2+}$ endógenos, proporciona a mineralização do tecido, então denominado "tecido duro". Por causa dessa alta alcalinidade, semelhante à de uma solução de hidróxido de cálcio, também se atribui ao MTA a característica de tornar o meio inóspito ao crescimento de bactérias $[2,3]$. Contudo, o MTA possui baixa resistência mecânica à compressão e este é um importante fator a ser considerado quando um material reparador é colocado em uma cavidade que irá sofrer pressão oclusal, como preenchimento de canal do dente [2] ou quando usado como base restauradora. Outra limitação do MTA diz respeito ao seu longo tempo de pega $[3,4]$. Quando usado como um material para preenchimento de canal o rápido endurecimento deve também reduzir o risco de deslocamento do material e contaminação. O endurecimento do material tão logo colocado na cavidade permite menor tempo de contato deste com tecidos vitais [2].

Outro biomaterial de destaque é o cimento ósseo, também conhecido como cimento ortopédico ou acrílico, polimetilmetacrilato (PMMA). O PMMA um material composto pela mistura de um monômero (líquido) e um polímero (pó), os quais são unidos pelo processo de polimerização. Atualmente, todos os cimentos ósseos no mercado são baseados quimicamente na mesma substância, o metilmetacrilato (MMA), um éster do ácido metacrílico. O PMMA pode servir como um espaçador, como um veículo de liberação de antibióticos e também pode ser colocado para eliminar o espaço morto. O cimento ortopédico é usado na cirurgia do quadril, na cirurgia da coluna vertebral, ombro, cotovelo, etc. É o principal componente para fixar as próteses dentro dos ossos [5,6]. Adicionando o monômero ao polímero, seus componentes são misturados e o processo de polimerização é então iniciado ocorrendo a auto-cura. Em temperatura ambiente $\left(23 \pm 1{ }^{\circ} \mathrm{C}\right)$, a polimerização do monômero somente pode ser iniciada na presença de radicais livres os quais são produzidos durante a reação do iniciador peróxido de benzoíla e do acelerador N,N-Dimetil p-toluidino. $\mathrm{O}$ processo transforma o líquido viscoso inicial em um material deformável macio e finalmente em um cimento, endurecendo-se rapidamente $(6-8 \mathrm{~min})$ com um aumento associado na temperatura. $\mathrm{O}$ processo de polimerização é uma reação exotérmica que libera uma grande quantidade de calor, fazendo com que temperaturas superiores a $90{ }^{\circ} \mathrm{C}$ sejam atingidas dentro do corpo humano. Estas temperaturas são prejudiciais às células em contato com o cimento, causando necrose do tecido ósseo ao redor da região do implante. Além disso, a necrose química pode ser atribuída também à liberação de monômero nãoreagido. A alta velocidade de reação do polímero também pode ocasionar queda da resistência mecânica do material, associada à má homogeneização durante o preparo, bem como a incorporação de ar. Na fixação de próteses o cimento atua como homogeneizador e amortecedor de altas tensões, principalmente de compressão. Falhas deste material podem ocasionar a soltura de próteses conduzindo inevitavelmente a novas cirurgias $[5,6]$.

Neste contexto, um novo biomaterial a base de cimento de aluminato de cálcio (CAC) tem sido estudado visando preservar as propriedades positivas e aplicações clínicas do MTA e PMMA permitindo que suas aplicações possam ser extendidas, superando as limitações apresentadas por esses materiais [7-9]. O cimento de aluminato de cálcio comercial é composto principalmente pelas fases $\mathrm{CA}(\mathrm{CaO}$. $\left.\mathrm{Al}_{2} \mathrm{O}_{3}\right)$ e $\mathrm{CA}_{2}\left(\mathrm{CaO} .2 \mathrm{Al}_{2} \mathrm{O}_{3}\right)$ as quais são responsáveis pelo seu processo de endurecimento hidráulico [10]. A dissolução dessas fases em contato com a água promove a liberação dos íons $\mathrm{Ca}^{2+}, \mathrm{Al}(\mathrm{OH})_{4}^{-}$e $\mathrm{OH}^{-}$, o que é, devido a saturação da solução, seguido pela precipitação de hidratos de aluminato de cálcio, compostos por $\mathrm{CaO}, \mathrm{Al}_{2} \mathrm{O}_{3}$ e $\mathrm{H}_{2} \mathrm{O}$ e hidróxido de alumínio [11]. Rotas de produção de cimento de aluminato de cálcio foram estudadas [12] com a finalidade de conhecer os processos de síntese e indicar a melhor rota para a produção isolada de cada uma das diferentes fases do CAC e do nível de impureza presente no material e assim desenvolver uma composição mais adequada, potencializando o uso do CAC nas áreas pretendidas.

Dessa forma, uma vez otimizada a produção de cada uma das fases do CAC, este trabalho tem como objetivo apresentar a sua caracterização quanto à temperatura de reação de hidratação; trabalhabilidade/tempo de presa e quanto a influência sobre $\mathrm{pH}$, condutividade iônica e concentração de sólidos da água e diferentes soluções simuladoras de fluido corporal quando em contato com as fases.

\section{MATERIAIS E MÉTODOS}

Diferentes fases de cimento de aluminato de cálcio foram sintetizadas previamente por meio da calcinação de $\mathrm{Al}_{2} \mathrm{O}_{3} \mathrm{e}$ $\mathrm{CaO}$ utilizando-se um forno elétrico (Lindberg Blue, USA), em diferentes temperaturas com patamar de uma hora. Os materiais usados na síntese das fases foram alumina calcinada A1000SG (99,8\% $\mathrm{Al}_{2} \mathrm{O}_{3}$, Almatis) e óxido de cálcio (100\% $\mathrm{CaO}$, Vetec). As matérias-primas foram adicionadas em um frasco de polietileno de alta densidade (HDPE) contendo esferas de alumina na proporção 5:1 (esferas:pó). O frasco foi mantido em um moinho de bolas durante uma hora para 
a homogeneização da mistura. As quantidades utilizadas das diferentes matérias-primas bem como as condições de queima foram ajustadas visando à produção de $\mathrm{CAC}$ com diferentes fases isoladas: $\mathrm{CA}\left(\mathrm{CaO} \cdot \mathrm{Al}_{2} \mathrm{O}_{3}\right), \mathrm{CA}_{2}\left(\mathrm{CaO} \cdot 2 \mathrm{Al}_{2} \mathrm{O}_{3}\right), \mathrm{C}_{3} \mathrm{~A}$ $\left(3 \mathrm{CaO} \cdot \mathrm{Al}_{2} \mathrm{O}_{3}\right)$ e $\mathrm{C}_{12} \mathrm{~A}_{7}\left(12 \mathrm{CaO} .7 \mathrm{Al}_{2} \mathrm{O}_{3}\right)$. A determinação da proporção entre as matérias-primas foi realizada com base no diagrama de equilíbrio para o sistema binário $\mathrm{Al}_{2} \mathrm{O}_{3}-\mathrm{CaO}$ [12]. Os materiais sintetizados foram desaglomerados em almofariz de porcelana e a produção das diferentes fases foi confirmada por difração de raios $\mathrm{X}$ empregando-se um difratômetro Shimadzu XRD-6000 e radiação $\mathrm{Cu}-\mathrm{k} \alpha(\lambda=1,54439 \AA)$. Posteriormente, as fases isoladas foram submetidas a um processo de moagem a seco durante $17 \mathrm{~h}$ com moinho de bolas e esferas de alumina na proporção 5:1 (esferas:pó) [12]. Após a moagem, as fases sintetizadas foram caracterizadas quanto à temperatura de reação de hidratação; trabalhabilidade e tempo de presa; $\mathrm{pH}$, solubilização de íons e dissolução em contato com água e diferentes soluções simuladoras de fluido corporal. O MTA-branco (Angelus, Brasil) e o cimento ósseo PMMA (Biomecânica, Brasil) foram usados como materiais de controle.

A preparação das suspensões aquosas das diferentes fases do CAC foi realizada adicionando água aos pós até a obtenção de uma massa moldável. A concentração de sólidos obtida foi próxima ao material de controle (MTA) no caso das fases $\mathrm{CA}$ e $\mathrm{CA}_{2}$, enquanto para as fases $\mathrm{C}_{3} \mathrm{~A}$ e $\mathrm{C}_{12} \mathrm{~A}_{7}$ foi necessário a adição de um maior teor de água devido ao aquecimento da suspensão (Tabela I). Para os ensaios de avaliação do aumento de temperatura, as suspensões aquosas dos materiais foram preparadas efetuando-se a mistura da água ao pó a temperatura ambiente seguida da adição em um suporte o qual permitiu o controle da temperatura em $37^{\circ} \mathrm{C}$. A temperatura de reação de hidratação foi avaliada por meio da inserção de um termopar nas suspensões sendo as medidas imediatamente iniciadas. O termopar foi conectado a um equipamento medidor de $\mathrm{pH}$ com sensor de temperatura individual (MA 522/E, Marconi) com aquisição automática de dados (1 dado/s). No caso do material controle, PMMA, o monômero líquido foi misturado ao pó e o sensor de temperatura imediatamente inserido.

Suspensões aquosas das diferentes fases sintetizadas

Tabela I - Concentração de sólidos usada na preparação de suspensões aquosas dos materiais.

[Table I - Solid concentration used for preparating the materials aqueous suspensions.]

\begin{tabular}{cc}
\hline Material & Concentração de sólidos (\%-p) \\
\hline MTA & 65 \\
$\mathrm{CA}$ & 63 \\
$\mathrm{CA}_{2}$ & 67 \\
$\mathrm{C}_{3} \mathrm{~A}$ & 59 \\
$\mathrm{C}_{12} \mathrm{~A}_{7}$ & 50 \\
\hline
\end{tabular}

também foram preparadas com concentrações de sólidos apresentadas na Tabela I e utilizadas para os ensaios de trabalhabilidade e tempo de presa. Para esses ensaios as suspensões foram vertidas em um porta amostra (180 $\mathrm{mL}$ ) para medidas quanto ao tempo de endurecimento inicial (trabalhabilidade) e final (presa) usando um equipamento de registro automático Vicat (Vicatronic E044-Solotest), mantendo-se a temperatura ambiente em aproximadamente $37{ }^{\circ} \mathrm{C}$. Os ensaios para as medidas de tempo de endurecimento consistiram em medir a distância de penetração da agulha na amostra a cada 3 ou 4 min para aquelas contendo $\left(\mathrm{C}_{3} \mathrm{~A}\right.$ ou $\left.\mathrm{C}_{12} \mathrm{~A}_{7}\right)$ e $(\mathrm{CA}$ ou $\mathrm{CA}_{2}$ ), respectivamente. As medidas foram realizadas até que a agulha não conseguisse mais penetrar na amostra, indicando seu endurecimento final. Suspensões aquosas das diferentes fases sintetizadas também foram preparadas de acordo com a Tabela I e usadas na preparação de amostras (10 mm de diâmetro x $5 \mathrm{~mm}$ de altura) como apresentado na Fig. 1. Após a preparação, as suspensões foram vertidas nos moldes, os quais foram mantidos em estufa a $37^{\circ} \mathrm{C}$ durante $24 \mathrm{~h}$ em ambiente saturado. Após este tempo, as amostras foram desmoldadas e mantidas a $37^{\circ} \mathrm{C}$ em ambiente saturado durante 7 dias. Após esse período foram colocadas em acetona para parada da reação de hidratação e usadas para avaliação do $\mathrm{pH}$, solubilização de íons (condutividade iônica) e medidas de dissolução.

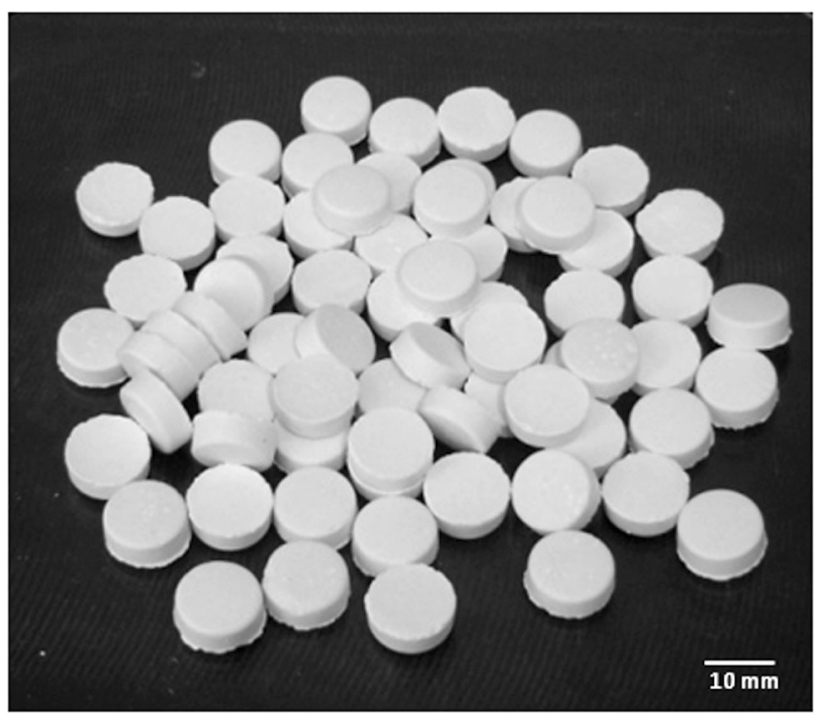

Figura 1: Amostras produzidas a partir da moldagem de suspensões de fases de cimento de aluminato de cálcio.

[Figure 1: Samples produced by casting the suspensions of calcium aluminate cement phases.]

Os ensaios consistiram em medidas de $\mathrm{pH}$ e condutividade iônica em $50 \mathrm{~mL}$ de água ou diferentes soluções simuladoras de fluido corporal (SBF) em contato com as amostras das fases, em função do tempo (até 21 dias). Foram utilizados um equipamento medidor de $\mathrm{pH}$ e um condutivímetro, ambos com sensor de temperatura individual (MA 522/E e MA 521/E, Marconi). A preparação das soluções de fluido corporal simulada (SBF) seguiu 
Tabela II - Reagentes usados na preparação de um litro de solução de fluido corporal simulado de acordo com Kokubo. [Table II - Reagents used for preparating the simulated body fluid solution according to Kokubo.]

\begin{tabular}{clll}
\hline Ordem & Reagente & SBF 1,0 & SBF 1,5 \\
\hline$\# 0$ & Água & $750 \mathrm{~mL}$ & $750 \mathrm{~mL}$ \\
$\# 1$ & $\mathrm{NaCl}$ & $7,996 \mathrm{~g}$ & $11,994 \mathrm{~g}$ \\
$\# 2$ & $\mathrm{NaHCO}_{3}$ & $0,350 \mathrm{~g}$ & $0,525 \mathrm{~g}$ \\
$\# 3$ & $\mathrm{KCl}$ & $0,224 \mathrm{~g}$ & $0,336 \mathrm{~g}$ \\
$\# 4$ & $\mathrm{~K}_{2} \mathrm{HPO}_{4}$ & $0,174 \mathrm{~g}$ & $0,288 \mathrm{~g}$ \\
$\# 5$ & $\mathrm{MgCl}_{2} \cdot 6 \mathrm{H}_{2} \mathrm{O}$ & $0,305 \mathrm{~g}$ & $0,458 \mathrm{~g}$ \\
$\# 6$ & $\mathrm{HCl} \mathrm{1M}_{\# 7}$ & $40 \mathrm{~mL}$ & $60 \mathrm{~mL}$ \\
$\# 8$ & $\mathrm{CaCl}_{2} \cdot 2 \mathrm{H}_{2} \mathrm{O}$ & $0,368 \mathrm{~g}$ & $0,507 \mathrm{~g}$ \\
$\# 9$ & $\mathrm{Na}_{2} \mathrm{SO}_{4}$ & $0,071 \mathrm{~g}$ & $0,107 \mathrm{~g}$ \\
\hline & $\left.\mathrm{CH}_{2} \mathrm{OH}\right)_{3} \mathrm{CNH}$ & $6,057 \mathrm{~g}$ & $9,086 \mathrm{~g}$ \\
\hline 10 & $\mathrm{HCl} 1 \mathrm{M}$ & Necessária & Necessária \\
& & para ajuste & para ajuste \\
do pH=7,25 & do pH=7,25 \\
\hline
\end{tabular}

Tabela III - Reagentes usados na preparação de um litro de solução de fluido corporal simulado de acordo com Rigo.

[Table III - Reagents used for preparating the simulated body fluid solution according to Rigo.]

\begin{tabular}{|c|c|c|}
\hline Ordem & Reagente & SBF 1,5 \\
\hline$\# 0$ & Água & $400 \mathrm{~mL}$ \\
\hline$\# 1$ & $\mathrm{NaCl}$ & $11,992 \mathrm{~g}$ \\
\hline$\# 2$ & $\mathrm{KCl}$ & $0,335 \mathrm{~g}$ \\
\hline$\# 3$ & $\mathrm{~K}_{2} \mathrm{HPO}_{4}$ & $0,261 \mathrm{~g}$ \\
\hline$\# 4$ & $\mathrm{NaHCO}_{3}$ & $0,529 \mathrm{~g}$ \\
\hline$\# 5$ & $\mathrm{Na}_{2} \mathrm{SO}_{4}$ & $0,107 \mathrm{~g}$ \\
\hline$\# 6$ & $\mathrm{HCl} 0,1 \mathrm{M}$ & $15 \mathrm{~mL}$ \\
\hline$\# 7$ & $\mathrm{CaCl}_{2} \cdot 2 \mathrm{H}_{2} \mathrm{O}$ & $0,551 \mathrm{~g}$ \\
\hline$\# 8$ & $\mathrm{MgCl}_{2} \cdot 6 \mathrm{H}_{2} \mathrm{O}$ & $0,458 \mathrm{~g}$ \\
\hline$\# 9$ & $\begin{array}{l}\mathrm{HCl} \mathrm{0,1M} \\
{\left[\left(\mathrm{CH}_{2} \mathrm{OH}\right)_{3} \mathrm{CNH}_{2}\right] 0,05 \mathrm{M}}\end{array}$ & $\begin{array}{l}\text { Necessária } \\
\text { para ajuste do } \\
\mathrm{pH}=7,25\end{array}$ \\
\hline
\end{tabular}

procedimentos descritos [13, 14], como apresentado nas Tabelas II e III, respectivamente. A solução de Kokubo foi preparada utilizando-se uma quantidade dos reagentes a fim de reproduzir a concentração de íons ( $\mathrm{mM}$ ) inorgânicos presentes no plasma humano (SBF 1) ou em uma proporção 1,5 vezes superior (SBF 1,5) a fim de acelerar a formação de apatita. A solução de Rigo foi preparada apenas na proporção de 1,5 .

Amostras também foram colocadas em contato com 50 $\mathrm{mL}$ de água ou de solução simuladora de fluido corporal (Kokubo 1,0). A cada dois dias, as pastilhas foram secas e pesadas a fim de avaliar a dissolução das amostras em função do tempo (até 34 dias).

\section{RESULTADOS E DISCUSSÃO}

\section{Temperatura de reação de hidratação}

Durante a hidratação das fases de cimento de aluminato de cálcio, certa quantidade de calor é liberada fazendo com que a temperatura da pasta de cimento aumente (reação exotérmica) [15]. Dessa forma, por meio da introdução de um termopar na pasta de ligante hidráulico, o aumento de temperatura pode ser detectado e usado como indicativo da reação dos cimentos com a água, constituindo-se assim, numa técnica muito simples para acompanhamento da evolução da reação com o tempo. Os resultados quanto à avaliação da temperatura de hidratação das fases sintetizadas são apresentados na Fig. 2, sendo o MTA e o PMMA usados como materiais de controle.

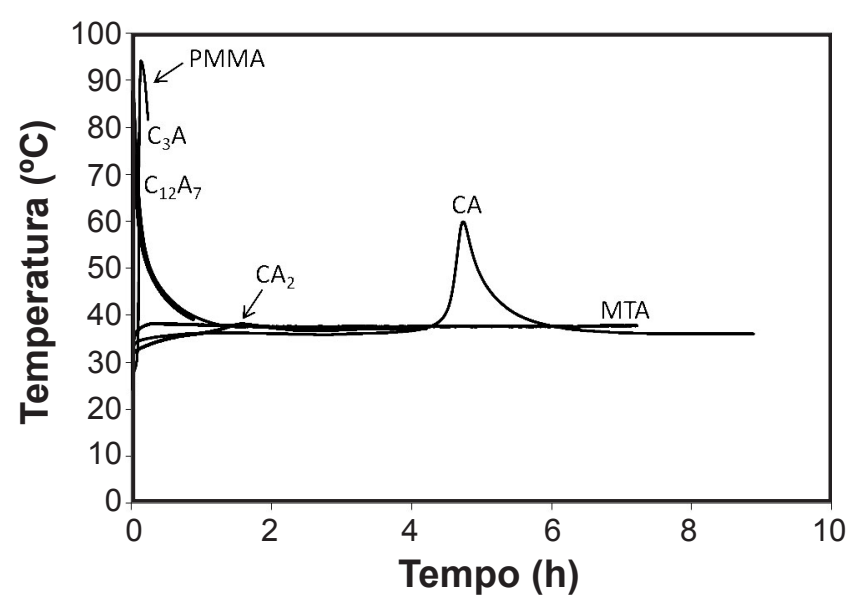

Figura 2: Variação da temperatura de suspensões aquosas de fases de cimento de aluminato de cálcio em função do tempo, usando o MTA e PMMA como materiais de controle.

[Figure 2: Temperature as a function of the time for aqueous suspensions of calcium aluminate cement phases, taking MTA and PMMA as the control materials.]

As fases $\mathrm{C}_{3} \mathrm{~A}_{\text {e }} \mathrm{C}_{12} \mathrm{~A}_{7}$ apresentaram uma grande elevação de temperatura, imediatamente após a adição dos pós a água. Isso ocorreu devido as fases mais ricas em $\mathrm{CaO}$ apresentarem grande afinidade pela água liberando uma grande quantidade de calor [12]. As outras fases apresentaram menor aumento de temperatura, principalmente a fase $\mathrm{CA}_{2}$ mais rica em $\mathrm{Al}_{2} \mathrm{O}_{3}$. Por outro lado, para o MTA não foi verificado aumento de temperatura dentro de todo o tempo do ensaio indicando uma cinética lenta de hidratação deste ligante e longo tempo de pega. Dessa forma, baseado no aumento da temperatura indicativo da cinética de reação, é esperado que as fases do cimento aluminoso apresentem um comportamento mais adequado para aplicações odontológicas uma vez que endurecem mais rapidamente quando comparadas ao MTA. Entretanto, quando se considera a temperatura alcançada, as fases com exceção da fase $\mathrm{CA}_{2}$ mostraram-se prejudiciais aos tecidos. O tecido ósseo é sensível ao aquecimento próximo a $47^{\circ} \mathrm{C}$, e temperatura em torno de $60^{\circ} \mathrm{C}$ ou superior resulta em paralisação permanente do fluxo sangüíneo e necrose 
do tecido ósseo [5]. Como visto, o biomaterial de maior uso na fixação de próteses, consolidação de fraturas ou reconstrução de defeitos ósseos, o PMMA, é preparado por meio de um processo de polimerização o qual libera uma grande quantidade de calor. De fato, temperatura superior a $90{ }^{\circ} \mathrm{C}$ foi observada para este material (Fig. 2). Dessa forma, a fase $\mathrm{CA}_{2}$ também se apresenta como a mais indicada para aplicações em ortopedia uma vez que não desenvolvem grande aumento de temperatura durante o seu processo de hidratação. O desenvolvimento de composições com baixa temperatura de reação é fundamental para evitar a necrose do tecido ósseo ao redor da região do implante [5]. Além disso, cabe ressaltar que estudos ainda são necessários visando aumento da resistência mecânica das fases de CAC visando sua aplicação em Ortopedia. A incorporação de compostos de reforço como partículas ou fibras pode auxiliar na dissipação do calor gerado durante a hidratação do cimento.

\section{Trabalhabilidade e tempo de presa}

Os resultados das medidas da distância de penetração da agulha nas amostras em função do tempo envolvidos nos ensaios de trabalhabilidade e tempo de presa são apresentados na Fig. 3.

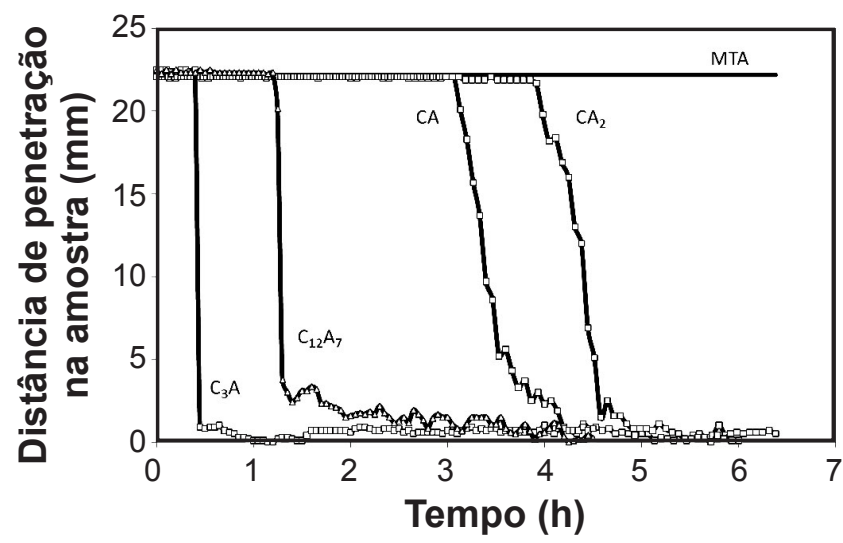

Figura 3: Avaliação da pega de suspensões aquosas de fases de cimento de aluminato de cálcio em função do tempo, usando o MTA como material de controle.

[Figure 3: Evaluation of setting time of aqueous suspensions of calcium aluminate cement phases, taking MTA as the control material.]

As fases sintetizadas apresentaram trabalhabilidade e tempo de presa que variaram conforme a sequência: $\mathrm{CA}_{2}$ $>\mathrm{CA}>\mathrm{C}_{12} \mathrm{~A}_{7}>\mathrm{C}_{3} \mathrm{~A}$. A diferença imposta pelo processo de hidratação das diferentes fases anidras está relacionada com a quantidade relativa entre as concentrações de $\mathrm{Ca}^{2+}$ e $\mathrm{Al}(\mathrm{OH})_{4}^{-}$que cada uma delas pode gerar em solução. Consequentemente, a concentração dos íons $\mathrm{Ca}^{2+} \mathrm{e} \mathrm{Al}(\mathrm{OH})_{4}$ em solução permitirá e ditará a fase de hidrato que será formada. A relação entre as concentrações desses íons em solução é convencionalmente representada por C/A que expressa a quantidade de cálcio e de alumínio na forma de $\mathrm{CaO}$ e $\mathrm{Al}_{2} \mathrm{O}_{3}$, respectivamente [16]. Sendo assim, o valor de C/A determina qual hidrato será formado em uma determinada temperatura. Para a temperatura de $25{ }^{\circ} \mathrm{C}$ e relação entre $\left[\mathrm{Ca}^{2+}\right] \mathrm{e}\left[\mathrm{Al}(\mathrm{OH})_{4}^{-}\right]$próximas a 1 , a formação do hidrato $\mathrm{CAH}_{10}$ é favorecida. Com o aumento dessa relação, ou seja, para maiores teores de $\mathrm{Ca}^{2+}$, passa-se a favorecer a formação de $\mathrm{C}_{2} \mathrm{AH}_{8}$. Já para maiores concentrações de $\mathrm{Al}(\mathrm{OH})_{4}^{-}$é predominante a formação de $\mathrm{AH}_{3}$ [16]. Os diferentes tipos de hidratos formados a partir do processo de hidratação do CAC diferem quanto ao grau de solubilidade, na seguinte ordem $\mathrm{C}_{3} \mathrm{AH}_{6}<\mathrm{C}_{2} \mathrm{AH}_{8}<\mathrm{CAH}_{10}<\mathrm{AH}_{3}$. A formação do tipo menos solúvel (mais rico em cálcio) é favorecida com o aumento do cálcio disponível para reação ou pelo aumento da temperatura. Isso implica numa alta velocidade de precipitação reduzindo o tempo de pega do cimento. Um raciocínio semelhante pode ser aplicado para se entender o atraso no tempo de pega quando o hidrato favorecido é o $\mathrm{AH}_{3}$, que inicialmente tem uma solubilidade maior que os demais hidratos e por esse motivo requer um maior período de indução.

O comportamento verificado neste trabalho quanto à trabalhabilidade e tempo de presa é compatível com o apresentado acima para as diferentes fases estudadas, uma vez que quanto maior o teor de $\mathrm{CaO}$, mais rápido é o endurecimento do cimento e menor é o tempo de presa como verificado para a fase $\mathrm{C}_{12} \mathrm{~A}_{7}$ e principalmente a $\mathrm{C}_{3} \mathrm{~A}$. Isso ocorre porque fases anidras mais ricas em cálcio, quando hidratadas, favorecem a formação de hidratos menos solúveis. Assim, o estágio de precipitação dos hidratos é acelerado. Tal estágio do processo de hidratação de ligantes hidráulicos é o responsável pelo travamento da estrutura, endurecimento do material e ganho de resistência mecânica. Por outro lado, a fase $\mathrm{CA}_{2}$ apresentou o maior tempo de pega devido ao menor teor de $\mathrm{CaO}$. $\mathrm{O}$ resultado para o MTA confirmou o longo tempo de pega indicado nos ensaios de temperatura, o que consiste em uma das suas principais desvantagens na aplicação em endodontia. O material reparador deve apresentar trabalhabilidade suficientemente grande para permitir a sua adequada aplicação e um curto tempo de pega, uma vez o rápido endurecimento deve reduzir o risco de deslocamento do material e contaminação $[2,17]$. O tempo de pega curto também facilita a dinâmina do atendimento clínico uma vez que passos subseqüentes a aplicação do material reparador, como o selamento do canal radicular, podem ser realizados na mesma sessão.

\section{pH, solubilização de íons e dissolução}

Os resultados das medidas de $\mathrm{pH}$ e condutividade iônica em água e nas soluções SBF são apresentados nas Figs. 4 e 5 , respectivamente.

Os resultados das medidas de dissolução das amostras em água e na solução SBF Kokubo 1,0 são apresentados na Fig. 6 .

A solubilização de íons $\mathrm{OH}^{-}$a partir das pastilhas é responsável pelo aumento do $\mathrm{pH}$ do meio enquanto a 

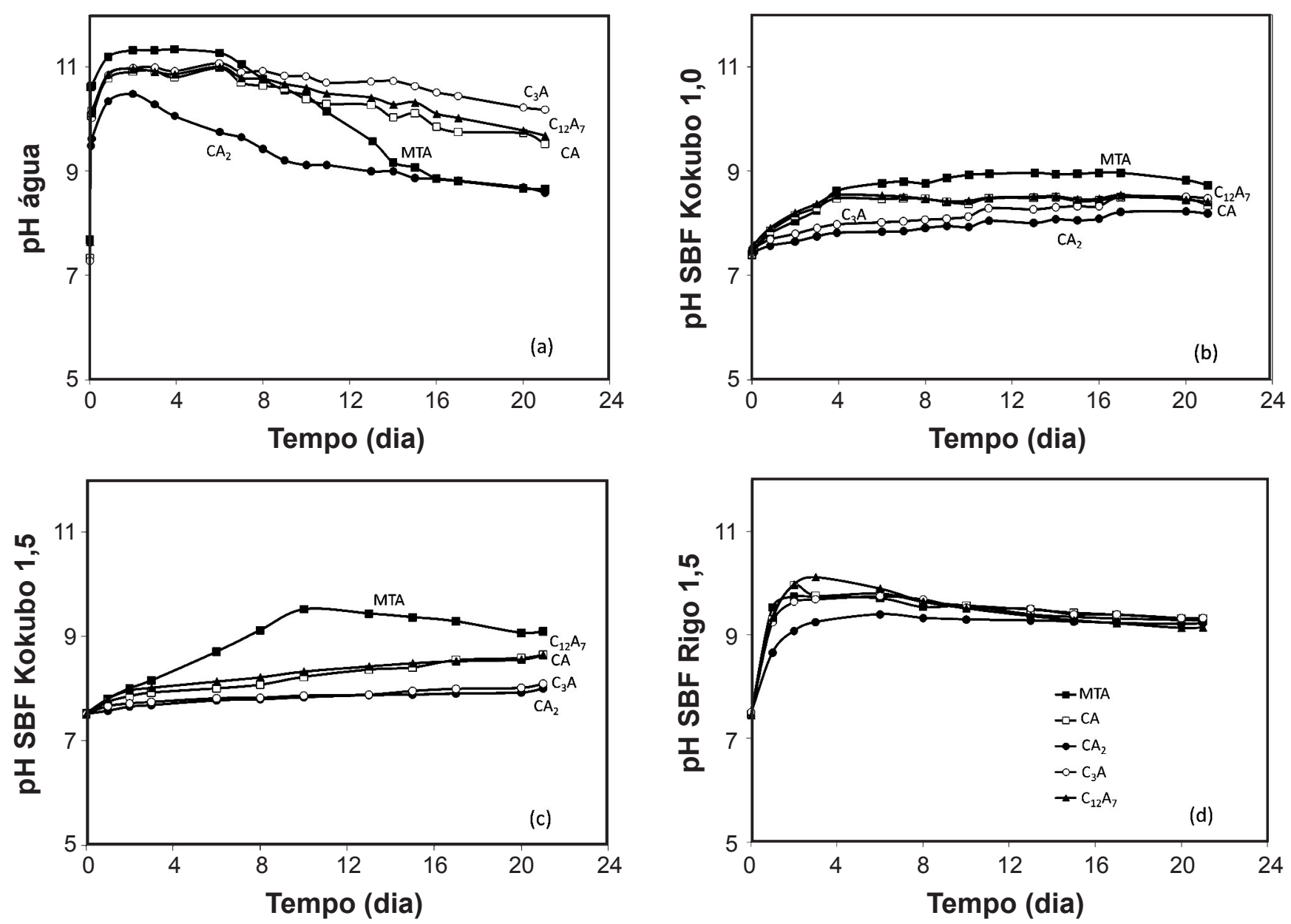

Figura 4: pH em função do tempo para (a) água, (b) solução SBF Kokubo 1,0, (c) solução SBF Kokubo 1,5 e (d) solução SBF Rigo 1,5; em contato com amostras endurecidas de MTA puro ou fases de cimento de aluminato de cálcio.

[Figure 4: pH as a function of the time for (a) water, (b) Kokubo 1.0 SBF (simulated body fluid), (c) Kokubo 1.5 SBF and (d) Rigo 1.5 SBF; in contact with set samples of plain MTA or calcium aluminate cement phases.]

liberação de íons $\mathrm{Ca}^{2+}$ e $\mathrm{OH}^{-}$é responsável pelo aumento da condutividade iônica. O principal componente do MTA endurecido responsável pela solubilização de íons é o hidróxido de cálcio que em contato com a água libera uma grande quantidade de íons $\mathrm{Ca}^{2+}$ e $\mathrm{OH}^{-}$no meio, o que pode ser associado ao significativo aumento inicial do $\mathrm{pH}$ (Fig. 4a) e principalmente da condutividade iônica (Fig. 5a) da água:

$$
\begin{aligned}
& 2\left(3 \mathrm{CaO} \cdot \mathrm{SiO}_{2}\right)+6 \mathrm{H}_{2} \mathrm{O} \rightarrow \\
& 3 \mathrm{CaO} \cdot 2 \mathrm{SiO}_{2} \cdot 3 \mathrm{H}_{2} \mathrm{O}+3 \mathrm{Ca}(\mathrm{OH})_{2} \\
& \mathrm{Ca}(\mathrm{OH})_{2}+\mathrm{H}_{2} \mathrm{O} \rightarrow \mathrm{Ca}^{2+}+\mathrm{OH}^{-}
\end{aligned}
$$

Pode-se observar que após aproximadamente 5 dias as medidas da amostra MTA endurecido em contato com a água indicaram uma condutividade de $1600 \mu \mathrm{S} / \mathrm{cm}$ (Fig. 5a) a qual esta associada à grande liberação de íons $\mathrm{Ca}^{2+}$. Após esse tempo foi verificado uma queda na condutividade o que pode ser atribuído a formação de carbonato de cálcio que induz o consumo dos íons $\mathrm{Ca}^{2+}$ e liberação de íons $\mathrm{H}^{+}$no meio, diminuindo também o pH (Fig. 4a):

$$
\begin{gathered}
\mathrm{CO}_{2}+\mathrm{H}_{2} \mathrm{O} \leftrightarrow \mathrm{H}^{+}+\mathrm{HCO}_{3}^{-} \\
\mathrm{Ca}^{2+}+\mathrm{HCO}_{3}^{-} \leftrightarrow \mathrm{CaCO}_{3}+\mathrm{H}^{+}
\end{gathered}
$$

Já as fases sintetizadas $\left(\mathrm{CA}, \mathrm{CA}_{2}, \mathrm{C}_{3} \mathrm{~A}\right.$ e $\left.\mathrm{C}_{12} \mathrm{~A}_{7}\right)$ após processo de hidratação e cura são formadas por hidratos de aluminato de cálcio e hidróxido de alumínio, como no exemplo:

$$
\begin{aligned}
& 3\left(\mathrm{CaO} \cdot \mathrm{Al}_{2} \mathrm{O}_{3}\right)+12 \mathrm{H}_{2} \mathrm{O} \rightarrow \\
& \mathrm{Ca}_{3}\left[\mathrm{Al}(\mathrm{OH})_{4}\right]_{2}(\mathrm{OH})_{4}+4 \mathrm{Al}(\mathrm{OH})_{3}
\end{aligned}
$$

A liberação dos íons $\mathrm{OH}^{-}$na água pode ser atribuída apenas as dissoluções dos hidratos de aluminato de cálcio, uma vez que o hidróxido de alumínio é insolúvel em água. Assim, quanto maior a quantidade de $\mathrm{OH}^{-}$presente no hidrato, maior será o $\mathrm{pH}$. Os hidratos formados a partir da fase anidra $\mathrm{CA}_{2}$ são menos ricos em $\mathrm{CaO}$ e portanto liberam menos íons $\mathrm{OH}^{-}$justificando o menor aumento de $\mathrm{pH}$ entre as fases (Fig. 4a). A liberação de íons cálcio também depende do tipo de hidrato formado a partir das diferentes fases anidras. Quanto mais rica em cálcio for a fase anidra, mais 

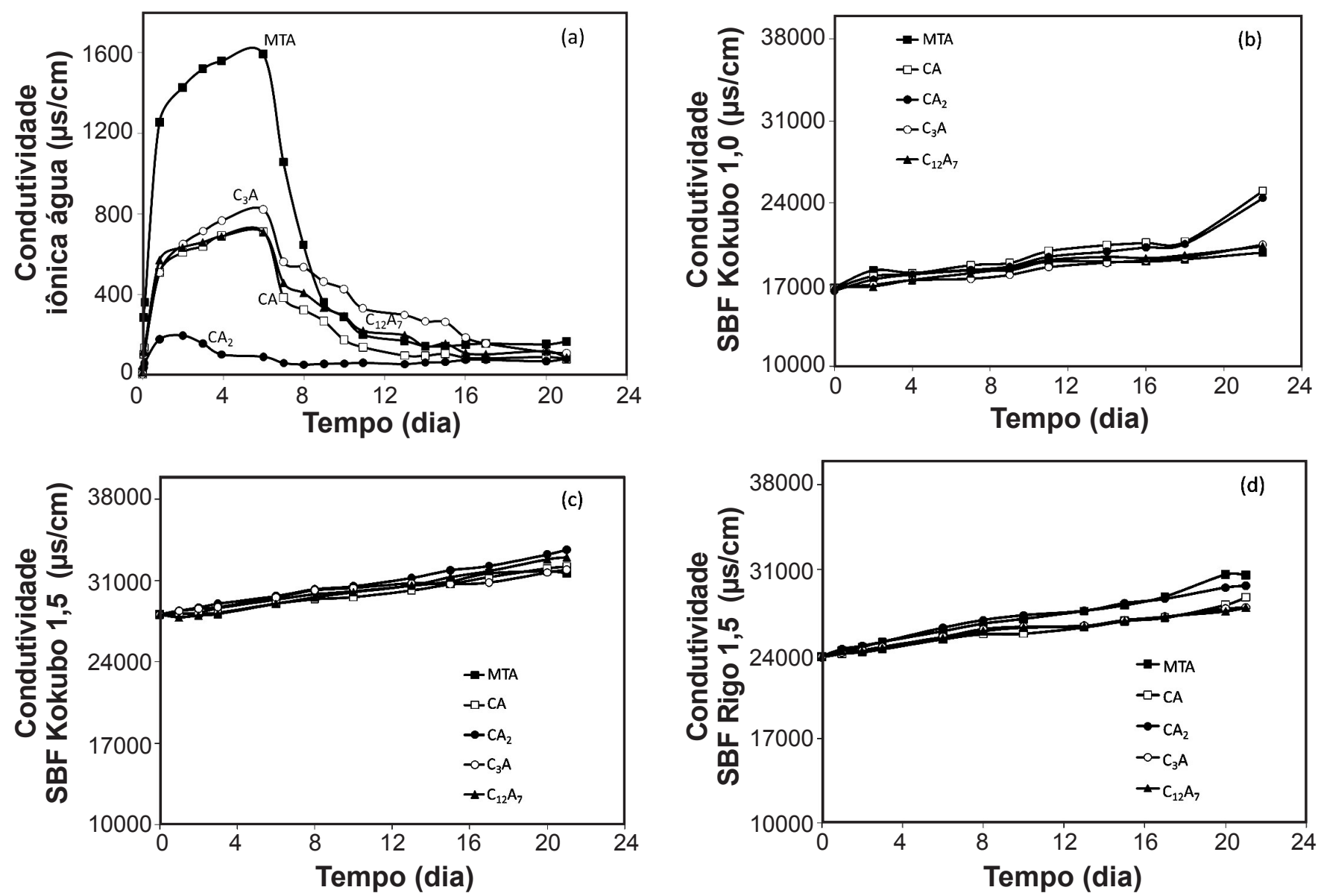

Figura 5: Condutividade iônica em função do tempo para (a) água, (b) solução SBF Kokubo 1,0, (c) solução SBF Kokubo 1,5 e (d) solução SBF Rigo 1,5; em contato com amostras endurecidas de MTA puro ou diferentes fases de cimento de aluminato de cálcio.

[Figure 5: Ionic conductivity as a function of the time for (a) water, (b) Kokubo 1.0 SBF (simulated body fluid), (c) Kokubo 1.5 SBF and (d) Rigo $1.5 \mathrm{SBF}$; in contact with set samples of plain MTA or calcium aluminate cement phases.]

cálcio para solubilização quando em contato com a água terá o hidrato de aluminato de cálcio presente na amostra endurecida. Dessa forma, a condutividade das fases também foi menor para a fase $\mathrm{CA}_{2}$ (Fig. 5a). Em relação ao pH, todos os materiais avaliados apresentaram a capacidade de alcalinizar o meio. O nível de alcalinização do meio em contato com um material determina a sua atividade antibacteriana. No caso da odontologia a reincidência de cáries é considerada o principal motivo que leva uma restauração a falhar. Deste modo, o emprego de agentes de cimentação que possuam propriedades antibacterianas é de considerável importância clínica [5]. Entretanto, a atividade antibacteriana é favorecida em condições de $\mathrm{pH}$ acima de 9,5 uma vez que bactérias não sobrevivem neste nível de $\mathrm{pH}$. Os resultados das medidas de $\mathrm{pH}$ para os materiais avaliados mostraram que tal condição é obtida facilmente em água mas não nas soluções de SBF já que se trata de meios tamponados visando o controle do $\mathrm{pH}$ do meio fisiológico (Fig. 4 b-d). Isso poderia ser confirmado comparando-se os níveis de $\mathrm{pH}$ alcançados nas soluções SBF de Kokubo e Rigo (Fig. 4 c e d). No método de preparo de Rigo, é utilizado um teor consideravelmente menor do tampão tris $\left[\left(\mathrm{CH}_{2} \mathrm{OH}\right)_{3} \mathrm{CNH}_{2}\right]$ sendo verificado, neste caso, níveis de $\mathrm{pH}$ consideravelmente maiores comparado as soluções de Kokubo. Por outro lado, os menores níveis de $\mathrm{pH}$ alcançados para as fases de CAC principalmente em soluções de SBF Kokubo indicam vantagens quanto as aplicações em ortopedia. Nestas aplicações, um requisito ideal do cimento para reparação óssea é a de desenvolver um $\mathrm{pH}$ neutro $(6,5-8,5)$ durante e depois da pega para evitar efeitos citotóxicos [5].

$\mathrm{Na}$ solução de fluido corporal o comportamento da condutividade foi muito diferente do verificado em água, principalmente para o MTA(Fig. 5). O fato da condutividade do MTA não aumentar tanto em SBF como ocorreu em água pode ser explicado pela reação dos íons $\mathrm{Ca}^{2+}$ liberados do hidróxido de cálcio, com íons fosfato $\left(\mathrm{PO}_{4}\right)^{3-}$ da solução SBF produzindo cristais de apatita sobre a superfície do MTA. De acordo com a literatura, a formação de apatita pode explicar a biocompatibilidade deste material [18, 19]. O SBF é uma solução simuladora do fluido corporal com concentrações inorgânicas similares ao do fluido extracelular humano, sendo uma solução metaestável contendo íons de cálcio e fosfato supersaturados, a fim de reproduzir a formação de apatita $[13,14]$. Os íons $\mathrm{Ca}^{2+}$ liberados do MTA são pouco solúveis em fluidos biológicos 

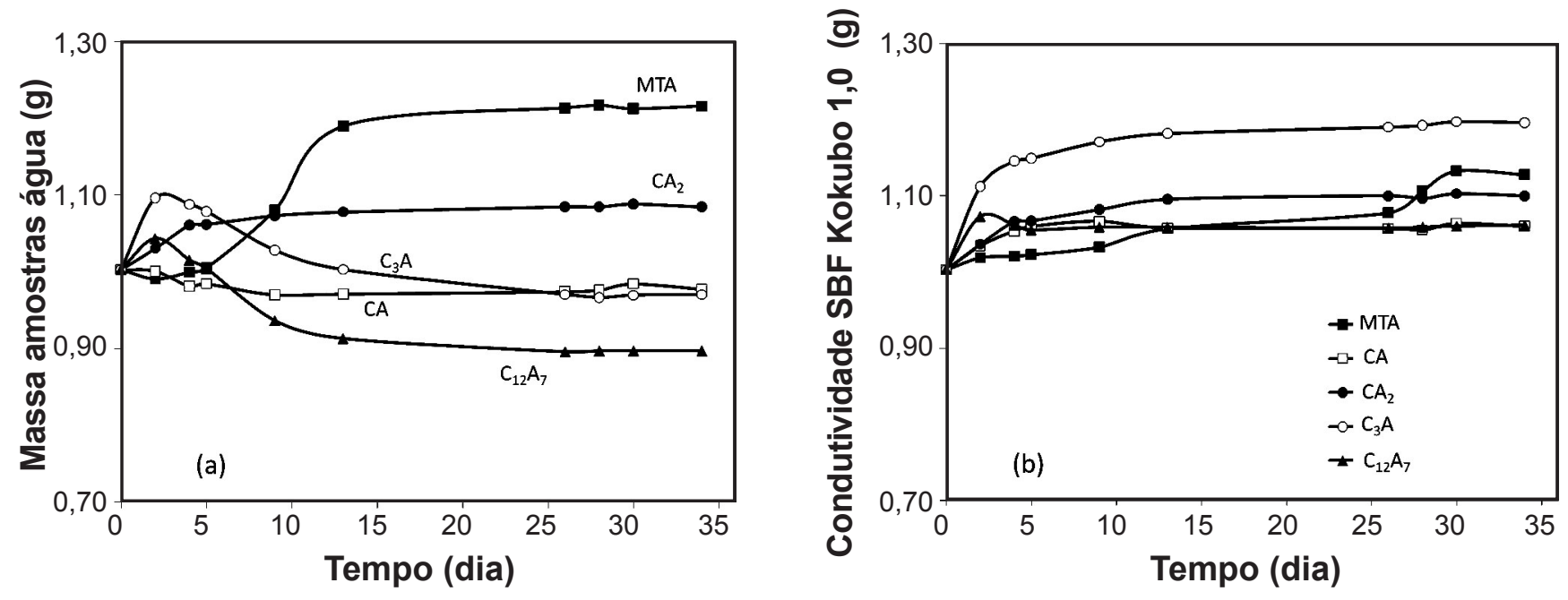

Figura 6: Massa das amostras endurecidas de MTA puro ou fases de cimento de aluminato de cálcio em contato com (a) água ou (b) solução SBF (Kokubo 1,0), em função do tempo.

[Figure 6: Mass of set samples of plain MTA or calcium aluminate cement phases in contact with (a) water or (b) Kokubo 1.0 SBF (simulated body fluid), as a function of the time.]

resultando na precipitação de hidroxiapatita (HA) [19].

$10 \mathrm{Ca}^{2+}+6\left(\mathrm{PO}_{4}\right)^{3-}+2(\mathrm{OH})^{-1} \rightarrow \mathrm{Ca}_{10}\left(\mathrm{PO}_{4}\right)_{6}(\mathrm{OH})_{2}$

Dessa forma, o consumo dos íons $\mathrm{Ca}^{2+}$ e $\mathrm{OH}^{-}$ provenientes do MTA para a formação de HA pode explicar o fato da condutividade não ter aumentado como observado na água (Fig. 5), além do menor valor de pH alcançado inicialmente em soluções SBF comparado a água (Fig. 4). Por outro lado, as fases do CAC também não apresentaram aumento da condutividade em SBF como verificado em água, o que indica a sua capacidade de apresentar formação de apatita ainda que menos significativo quando comparado ao MTA devido ao menor teor de íons cálcio liberado. Dessa forma, a presença de hidróxido de cálcio no MTA promove a dissociação de um maior teor de íons $\mathrm{Ca}^{2+} \mathrm{e}$ $\mathrm{OH}^{-}$os quais seriam inicialmente consumidos na formação de fases de fosfato de cálcio inibindo o aumento do $\mathrm{pH}$ e da condutividade iônica. Entretanto, como o consumo de $\mathrm{Ca}^{2+}$ é superior aos íons $\mathrm{OH}^{-}$, considerando a formação de hidroxiapatita (reação F), com o passar do tempo, os íons $\mathrm{OH}^{-}$ficam em excesso na solução resultando em um aumento mais lento do $\mathrm{pH}$ em SBF comparado a água. Isso pode ser verificado principalmente para o MTA em solução SBF Kokubo 1,5 (Fig. 4c). Já na presença de Kokubo 1,0 $\mathrm{o}$ aumento do $\mathrm{pH}$ é acelerado (Fig. 4b) e ainda mais rápido em SBF Rigo 1,5 (Fig. 4d). Esse comportamento pode ser entendido considerando que na solução SBF Rigo existe um teor significativamente menor do tampão Tris (Tabela III) e assim o pH é facilmente elevado pela liberação de íons $\mathrm{OH}^{-}$a partir dos materiais sem encontrar a resistência do tampão. Adicionalmente, cabe ressaltar que os ensaios para verificação quanto à dissolução das pastilhas em água e solução SBF revelaram que não ocorreu uma desintegração dos materiais (perda significativa de massa). Por outro lado, foi verificado ganho de massa principalmente para o MTA em água (Fig. 6a) e a fase $\mathrm{C}_{3} \mathrm{~A}$ em SBF (Fig. 6b) o que pode ser associado à maior porosidade ou tamanho de poros desses materiais permitindo absorção das soluções. De fato, o MTA apresenta uma considerável porosidade a qual é associada à pobre dispersão e coagulação das suas partículas [8].

\section{CONCLUSÕES}

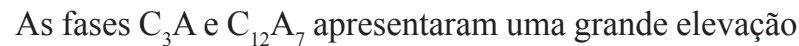
de temperatura durante a sua hidratação não sendo indicadas para aplicações em contato com tecidos vivos uma vez que pode causar a sua necrose. Por outro lado, menores aumentos de temperatura foram verificados para a fase $\mathrm{CA}$ e principalmente a fase $\mathrm{CA}_{2}$. A fase $\mathrm{CA}_{2}$ e principalmente a CA também apresentaram tempo de pega inferior ao MTA o que indica que essas fases podem superar uma das principais desvantagens apresentadas por esse produto na aplicação em endodontia. Além disso, podem ser combinadas com aditivos específicos para o ajuste do tempo ideal visando outras aplicações como na ortopedia assim como com outros compostos visando o aumento da sua resistência mecânica os quais podem auxiliar na dissipação do calor gerado pela fase CA. As fases $\mathrm{CA}_{2}$ e CA também apresentaram a capacidade de alcalinizar o meio, indicando sua capacidade de promover um ambiente antibacteriano e de interação com íons presentes na solução simuladora de fluido corporal (principalmente SBF Rigo), ao mesmo tempo em que não apresentam dissolução em contato com água ou SBF. Dessa forma, os resultados apresentados indicaram as fases CA e $\mathrm{CA}_{2}$ como as mais promissoras para dar continuidade aos estudos em busca de uma composição ideal para as aplicações pretendidas.

\section{AGRADECIMENTOS}

À FAPESP pelo suporte financeiro. 


\section{REFERÊNCIAS}

[1] M. E. M. Sayago, P. L. M. Pinto, J. P. P Collesi, Cirurgia perirradicular, in: H. P. Lopes, J. F. Siqueira Jr., "Endodontia: biologia e técnica", S. Paulo, Medsi 26 (1999) 577-597.

[2] M. Torabinejad, C. U. Hong, F. McDonald, T. R. Pitt Ford, Physical and chemical properties of a new root-end filling material, J. Endodontics 21 (1995) 349-353.

[3] H. W. Roberts, J. M. Toth, D. W. Berzins, D. G. Charlton, Mineral trioxide aggregate material use in endodontic treatment: A review of the literature, Dental Mater. 24 (2008) 149-164.

[4] E. A. Bortoluzzi, G. S. Araújo, J. M. Guerreiro Tanomaru, M. Tanomaru Filho, Marginal gingiva discoloration by gray MTA: a case report, J. Endodontics 33 (2007) 325-327.

[5] R. L. Oréfice, M. M. Pereira, H. S. Mansur, "Biomateriais: Fundamentos e Aplicações", Ed. Cultura Médica, Rio de Janeiro, RJ (2006).

[6] S. Saha, S. Pal, Mechanical properties of bone cement: A review, J. Biomedical Mater. Res. 18 (2004) 435-462.

[7] V. C. Pandolfelli, I. R. Oliveira, H. L. Rossetto, M. Jacobovitz, Composição a base de cimento aluminoso para aplicações em endodontia e produto cimentício obtido, Registro de Patente INPI 0704502-6, Universidade Federal de S. Carlos, SP (2007).

[8] I. R. de Oliveira, V. C. Pandolfelli, M. Jacobovitz, Chemical, physical and mechanical properties of a novel calcium aluminate endodontic cement, Int. Endodontic J. 43 (2010) 1069-1076.

[9] C. P. Burger, "Cimento de Aluminato de Cálcio - Uso em defeitos ósseos induzidos em fêmures de coelhos (Oryctolagus cuniculus)", Diss. Mestrado, Faculdade de Ciências Agrárias e Veterinárias, UNESP, Jaboticabal, SP (2010).
[10] K. M. Parker, J. H. Sharp, Refractory calcium aluminate cements, Brit.Ceram. Trans. J. 81 (1982) 35-42.

[11] C. Alt, L. Wong, C. Parr, Measuring castable rheology by exothermic profile, Refractories Appl. News 8 (2003) 158.

[12] T. L. Andrade, G. L. Santos, V. C. Pandolfelli, I. R. Oliveira, Otimização da síntese das fases de cimento de aluminato de cálcio, Cerâmica (2013) aceito.

[13] C. Ohtsuki, How to prepare the simulated body fluid (SBF) and its related solutions, proposed by Kokubo and his colleagues, http://mswebs.naist.jp/LABs/tanihara/ohtsuki/ $\mathrm{SBF} /$ index.html.

[14] C. C. G. Silva, E. C. S. Rigo, J. Marchi, A. H. A. Bressiani, J. C. Bressiani, Hydroxyapatite coating on silicon nitride surfaces using the biomimetic method, Mater. Res. 11 (2008) 47-50.

[15] C. M. George, Aspects of calcium aluminate cement hydration, in: "Refractories Symposium", The Am. Ceram. Soc., St. Louis, EUA (1994) 1-21.

[16] D. Sorrentino, F. Sorrentino, M. George, Mechanisms of hydration of calcium aluminate cements, Mater. Sci. Concrete IV, Ed.: J. Skalny, S. Mindess, Am. Ceram. Soc., Westerville, OH, EUA (1995) 41-90.

[17] J. Camilleri, The physical properties of accelerated Portland cement for endodontics use, Int. Endodontic J. 41 (2008) 151-157.

[18] M. Parirokh, S. Asgary, M. J. Eghbal, The long-term effect of saline and phosphate buffer solution on MTA: an SEM and EPMA investigation, Int. Endodontic J. 2 (2007) 81-86.

[19] N. K. Sarkar, R. Caicedo, P. Ritwik, R. Moiseyeva, I. Kawashima, Physicochemical basis of the biologic properties of mineral trioxide aggregate, J. Endodontics 31 (2005) 97-100.

(Rec. 06/02/2011, Rev. 23/05/2011, Ac. 24/05/2011) 